\title{
Management of STEMI
}

\author{
Swee Han Lim · Jeremy Wee $\cdot$ Venkataraman Anantharaman
}

Published online: 2 February 2013

(c) Springer Science+Business Media New York 2013

\begin{abstract}
There have been numerous advancements in the management of ST-elevation myocardial infarcts (STEMI) for the last few decades. Studies have shown an improvement of survival of STEMI due to increased use of perfusion therapy and recommended medications. The success of timely reperfusion therapy for STEMI patients would require close collaboration between pre-hospital care, emergency medicine, and cardiology departments to address the processes and professional issues in the various stages of management. This article reviews the latest management methods and issues, both pharmacological as well as reperfusion strategies. The different phases of emergency care for STEMIs were examined and various strategies for achieving rapid diagnosis and to improve door-to-balloon or door-to-needle time were discussed. Reperfusion methods of percutaneous coronary interventions and fibrinolysis were reviewed. Current evidence on adjuvant pharmacological therapies such as anti-platelet, heparin, anti-thrombin inhibitors, nitrate, $\beta$-blockers, and
\end{abstract}

S. H. Lim $(\bowtie) \cdot J$. Wee · V. Anantharaman

Department of Emergency Medicine, Singapore General

Hospital, Outram Road, Singapore 169608, Singapore

e-mail: gaelsh@sgh.com.sg

J. Wee

e-mail: jeremy.wee.c.p@sgh.com.sg

V. Anantharaman

e-mail: anantharaman@sgh.com.sg

S. H. Lim · J. Wee · V. Anantharaman

Yong Loo Lin School of Medicine, National University

of Singapore, Singapore 119077, Singapore

S. H. Lim · J. Wee · V. Anantharaman

Duke-NUS Graduate Medical School Singapore,

Singapore 169857, Singapore angiotensin-converting enzyme inhibitors were reviewed and examined.

Keywords Anti-platelet therapy · Percutaneous coronary intervention - Pre-hospital care - Fibrinolytics . Emergency department

\section{Introduction}

Since 1979, AMI can be diagnosed solely on the presence of unequivocal ECG changes (defined as development of abnormal, persistent Q or QS waves and evolving injury current) lasting longer than 1 day and/or unequivocal biomarker changes (initial rise and subsequent fall of the serum level) [1].

DeWood et al. [2] performed coronary angiograms on 322 AMI patients with ST elevations with subsequent progression to $\mathrm{Q}$ waves ( $\mathrm{Q}$ wave infarction or transmural infarction) and who were admitted within $24 \mathrm{~h}$ of infarction. Total coronary occlusion was found in $87 \%$ of patients who were evaluated within $4 \mathrm{~h}$ of the onset of symptoms and up to $65 \%$ of patients whose coronary angiograms were done 12-24 h after the onset of symptoms. DeWood repeated the study in patients within 1 week of non-Q wave myocardial infarction (MI). In total, coronary occlusion was found only in $26 \%, 37 \%$ and $42 \%$ of patients evaluated within $24 \mathrm{~h}$, between 24 and $72 \mathrm{~h}$, and between $72 \mathrm{~h}$ and 7 days after peak symptoms, respectively. The terms ' $\mathrm{Q}$ wave MI' and 'non-Q wave MI' were used [3]. With better understanding of the pathophysiology of AMI, a landmark study on the use of thrombolytic (later re-termed fibrinolytic) therapy was done. GISSI study group randomized patients with chest pain accompanied by ST elevation or depression of $1 \mathrm{~mm}$ or more in any limb 
lead of the ECG and/or $2 \mathrm{~mm}$ or more in any precordial lead and were admitted to CCU within $12 \mathrm{~h}$ from onset of symptoms with no contraindications to streptokinase. The streptokinase group had an improved mortality at 12 months compared with the control group (17.2 vs $19.0 \%, p=0.008$, relative risk 0.90). The study also showed that the shorter the time between the onset of pain and the infusion of streptokinase, the higher the chance of survival (relative risk of 0.89 and 0.87 respectively in the 0-3 and 3-6 h) [4].

It was thought that $\mathrm{Q}$ wave $\mathrm{MI}$ corresponded to transmural infarction and non-Q wave MI to subendocardial infarction. Studies using cardiac magnetic resonance imaging confirmed that $\mathrm{Q}$ waves are indicative of larger MI but not particularly useful in determining the extent of transmural and/or subendocardial infarction [5]. In the GUSTO (Global Utilization of Streptokinase and Tissue Plasminogen Activator for Occluded Coronary) Angiographic Sub-study, $20 \%$ of the AMI patients with ST segment did not progress to Q wave infarction. Hence, since 2000, the terms 'STEMI' (ST-segment elevation MI) and 'NSTEMI' (non-ST-segment elevation MI) have replaced ' $\mathrm{Q}$ wave $\mathrm{MI}$ ' and 'non-Q wave $\mathrm{MI}$ ', respectively. This change has been well received as the classification is very practical. Emergency reperfusion therapy has been shown to be only effective in STEMI and not NSTEMI [6].

When a patient presents with symptoms and signs of potential acute coronary syndrome (ACS), a diagnosis of STEMI is made when the ECG shows ST-segment elevation or new LBBB. The threshold values for ST-segment elevation consistent with STEMI are J-point elevation of $>1 \mathrm{~mm}$ in two or more contiguous leads except $\mathrm{V}_{2} \mathrm{~V}_{3}$. For $\mathrm{V}_{2} \mathrm{~V}_{3}$ a diagnosis of STEMI is made when men $<40$ years old have J-point elevations above $0.25 \mathrm{mV}$ $(2.5 \mathrm{~mm})$, for men $\geq 40$ years old the J-point elevation threshold is above $0.2 \mathrm{mV}(2.0 \mathrm{~mm})$ and for women above $0.15 \mathrm{mV}(1.5 \mathrm{~mm})$. However, ST elevation can also be present in several non-ACS conditions, e.g. benign early repolarization, acute pericarditis, and left ventricle aneurysm [7].

On the other hand, some patients with complete occlusion of coronary artery leading to massive MI do not have ST elevation on ECG, e.g. the presence of ST depression $>0.1 \mathrm{mV}$ in inferior lateral leads, coupled with ST elevation in aVR and/or $\mathrm{V}_{1}$ may suggest left main coronary artery occlusion or multi-vessels coronary artery obstruction [8].

$\mathrm{ST}$-segment depression in leads $\mathrm{V}_{1}-\mathrm{V}_{3}$ suggests a posterior infarction, especially when $\mathrm{T}$ wave is positive. The diagnosis can be confirmed by concomitant ST elevation in posterior leads $\mathrm{V}_{7}-\mathrm{V}_{9}$. $(\geq 0.1 \mathrm{mV})$.

\section{Emergency Care of STEMI}

\section{Pre-hospital Phase}

The management of STEMI is time sensitive. Earlier revascularization generally leads to reduction of infarct size, better myocardial function, and lower mortality [9•]. Any delay during the pre-hospital phase can occur from time of onset of the chest pain until presentation at the emergency department (ED). Studies have identified several factors associated with patients who have chest pain delaying in calling emergency medical services (EMSs), e.g. patient not recognizing that symptoms are acute coronary in nature, being alone at the time of presentation, female gender, or racial factors [10]. Public education campaigns have met with limited success as effects are often short-lasting, or even unintended, e.g. increases in ED attendance of non-coronary chest pain instead of ACS [11]. However, patients with coronary artery disease and their relatives should be educated on symptoms of MI and to activate EMS if the symptoms have persisted more than 20 min or are not relieved with sublingual nitrates.

The practice of obtaining pre-hospital 12-lead ECG when emergency ambulance crews (paramedics) attend to patients with chest pain and electronically transmitting these to the receiving ED before leaving the site will speed up the diagnosis, the door-to-balloon time (D2B) or door-to-needle time (D2N) [12]. Upon arrival of the patient at the ED, the emergency ambulance crew will also forward the hard copy of the ECG to the triage/ reception staff.

\section{Emergency Department Phase}

Potential delays in the ED can occur during the phases of registration, triage, doctor consultation, ECG, diagnosis making, consultation with a cardiologist, and having to make arrangements with the need for percutaneous intervention procedures. Decisions to review care processes for such patients have resulted in a changes which have significantly reduced D2B times from approximately $110 \mathrm{~min}$ to as low as 40-50 min (in the case of primary PCI), or D2N times from an average of $120 \mathrm{~min}$ to within $30 \mathrm{~min}$ for initiation of fibrinolytics when these were administered in the ED [13]. For patients with chest pain arriving at the ED via their own transportation, strategies are implemented to complete the initial ECG within 10 min of arrival and having these read by emergency physicians immediately.

If the ECG is diagnostic of STEMI, the patient will be managed under continuous ECG monitoring and a team of doctors, nurses and paramedical staff will attend to the patient. 
The following will be done almost concurrently:

(a) Obtain a targeted history, covering:

- Chest pain history including the time of onset and severity.

- Traditional risk factors for coronary arterial disease (CAD), such as prior history of MI, diabetes mellitus, hyperlipidemia, family history of premature CAD, cocaine and sildenafil (Viagra) use.

- Drug allergies.

(b) Perform a focused physical examination, including:

- Check vital signs.

- Elicit signs of cardiac failure-distended neck veins, third heart sounds, lung crepitations, murmurs (due to ventricular septal rupture or papillary muscle rupture).

- Feel all the peripheral pulses (this ritual is to acknowledge the very rare event of ST elevation in ECG due to aortic dissection occluding the coronary artery).

(c) Administer $300 \mathrm{mg}$ of aspirin and $180 \mathrm{mg}$ of ticagrelor/60 $\mathrm{mg}$ of prasugrel/ $600 \mathrm{mg}$ of clopidogrel if not contraindicated.

(d) If the patient is in pain, and systolic blood pressure is adequate, sublingual glyceryl tri-nitrate (GTN) may be administered. Intravenous morphine $1-2 \mathrm{mg}$ aliquots are given, titrated against the symptom (premedication with intravenous cyclizine or metoclopramide is commonly practiced).

(e) Inform patient of the diagnosis and the need for primary PCI, complications of the procedure (and fibrinolytic therapy if applicable) and obtain consent.

Activate the cardiac catheterization laboratory. Shave the groin area and prepare to transfer patient to cardiac catheterization laboratory.

PCI is the preferred reperfusion strategy of STEMI, [14•, 15.*] provided it can be performed with a D2B time of $<90$ min by a skilled operator (performing $>75$ PCIs per year) at a PCI capable centre (performing $>200$ PCIs a year, of which at least 36 are primary PCI for STEMI) [16••]. This is because PCI can be effective for a wider range of patients as compared to fibrinolytics (including patients with contraindications to fibrinolytics and uncertain STEMI diagnosis), a lower risk of intracranial bleeding complication and a higher initial coronary reperfusion rate. Those receiving hospitals that do not have PCI capabilities should have protocol to transfer the STEMI patients to a PCI center. The non-PCI hospitals should also collaborate with EMSs to initiate pre-hospital 12-lead ECG and protocol to send patients to the nearest PCI centre, bypassing those without [17]. If the paramedics and medical control are well trained and are able to analyse 12-lead ECG on site or transmit to medical facilities for confirmation, fibrinolytic therapy can be initiated in the prehospital setting to further reduce D2N time.

If the transfer is expected to be significantly delayed, fibrinolysis must be considered for the patients without contraindication(s) to fibrinolysis. How long should a STEMI patient who is eligible for fibrinolytics wait for PCI before the additional benefits of PCI are lost and fibrinolysis becomes the preferred option? A study suggests that an individualized approach should be adopted as the time recommended depends on the patient's age, infarction duration and location of STEMI. For example anterior STEMI with age $<65$ years is around $40 \mathrm{~min}$, non-anterior MI with age $>65$ years is around 170 min [18]. However, this is a post hoc analysis of a registry. This result must be interpreted with caution, and it will be confusing for ground staff to implement such a protocol. The 2012 European Society of Cardiology guidelines recommended that fibrinolysis should be considered if PCI cannot be performed within $120 \mathrm{~min}$ of first medical contact [19••].

Though fibrinolytics are well-proven to improve coronary blood flow, limit infarct size and improve survival, certain contraindications exist. The absolute contraindications include [4]:

(a) known intracranial haemorrhage at any time,

(b) ischaemic stroke in the preceding 6 months,

(c) presence of intracranial tumour, arterio-venous malformation or bleeding,

(d) major surgery or major trauma/head injury within the preceding 3 weeks,

(e) presence of active internal bleeding (excepting menstruation),

(f) gastrointestinal bleeding within the past month,

(g) bleeding diathesis,

(h) non-compressible punctures in the past $24 \mathrm{~h}$ (e.g. liver biopsy, lumbar puncture, subclavian puncture),

(i) high suspicion of aortic dissection,

(j) pericarditis,

(k) refractory to resuscitation,

(l) allergy to the intended thrombolytic or previous reaction to these agents,

(m) pregnancy.

Relative contraindications include:

(a) high blood pressure with at least two simultaneous readings $>180 / 110 \mathrm{mmHg}$,

(b) severe renal or liver disease,

(c) prolonged or traumatic CPR but successful resuscitation, 
(d) patients on chronic anti-coagulant therapy (e.g. warfarin).

A number of thrombolytic agents are available. The current recommended doses of some of the more commonly used thrombolytic agents are as follows:

(1) Streptokinase 1.5 million units (u) given intravenously over $60 \mathrm{~min}$ [4].

(2) $t$-PA (alteplase) $15 \mathrm{mg}$ initial IV bolus, followed by $0.75 \mathrm{mg} / \mathrm{kg}$ body weight (maximum $50 \mathrm{mg}$ ) over the next $30 \mathrm{~min}$ and then $0.5 \mathrm{mg} / \mathrm{kg}$ body weight (maximum $35 \mathrm{mg}$ ) over the remaining $60 \mathrm{~min}$.

(3) $T N K-t P A$ (tenecteplase) $0.5 \mathrm{mg} / \mathrm{kg}$ body weight as a bolus intravenous dose over 5 seconds (minimum dose $30 \mathrm{mg}$, maximum dose $50 \mathrm{mg}$ ).

(4) $r$-PA (reteplase) $2 \times 10 \mathrm{u}$ each over $2 \mathrm{~min}$ and given 30 min apart.

All thrombolytics are said to have almost equivalent efficacy in achieving vascular patency with small differences in bleeding and mortality rates. The drug and dose used will be dependent on availability and cost considerations-streptokinase a non-fibrin-specific fibrinolytic is approximately $1 / 10$ the cost of fibrin-specific agents. Administration of streptokinase may be associated with hypotension but severe allergic reaction is rare. Readministration of streptokinase should be avoided because of antibodies, which can impair its activity and increase the risk of allergic reactions. In a randomized study comparing tPA and unfractionated heparin (UFH) with streptokinase given IV, the tPA arm had 10 fewer deaths per 1,000 patients treated [20]. The mutant forms of tPA such as rPA and TNK-tPA (see above) are equivalent to tPA but easier to administer in the pre-hospital setting [21].

After initiation of fibrinolytic therapy, the patient should be transferred urgently to a PCI centre if there is evidence of failed fibrinolysis, i.e. recurrence of symptoms or STsegment elevation. Even if the fibrinolysis is successful, i.e. disappearance of chest pain, ST-segment resolution, appearance of reperfusion arrhythmia; routine early referral (within $24 \mathrm{~h}$ ) of patients for post-fibrinolysis angiography with subsequent PCI (if required) may reduce the rate of re-infarction and recurrent ischemia.

\section{Adjuvant therapy for STEMI patients before undergoing PCI or fibrinolysis}

Prior to angiography, such patients should receive double anti-platelet therapy with aspirin and an adenosine diphosphate (ADP)-receptor blocker, and a parenteral anticoagulant, as early as possible.
Anti-platelet Agents

(1) Aspirin should be administered early in the ED to all patients suspected of having ACS and who have no contraindications to aspirin. Aspirin irreversibly acetylates platelet cyclooxygenase. The second International Study of Infarct Survival [ISIS-2] trial [22] provides the strongest evidence that aspirin has been shown to independently reduce the mortality of AMI patients with or without fibrinolytic therapy by up to 23 and $42 \%$ respectively. The recommended first dose is $150-300 \mathrm{mg}$ of non-enteric-coated aspirin, and a lower dose 75-150 mg thereafter. Aspirin can be given intravenously in patients who cannot take it orally. The optimal intravenous dosage ranges from 80 to $151 \mathrm{mg}$.

(2) Other anti-platelet agents include the thienopyridines prodrug, e.g. ticlopidine, clopidogrel, prasugrel and ticagrelol. These inhibit the ADP dependent pathway of platelet activation. Ticlopidine produces its maximal effect after 8-11 days of repeated intake. It is associated with a risk of neutropenia and agranulocytosis. Clopidogrel, a ticlopidine analogue, is preferred over ticlopidine because of its rapid onset of action and safety profile. Administration of clopidogrel, $75 \mathrm{mg}$ daily, results in maximal platelet inhibition after 4-7 days. Clopidogrel in combination with aspirin is more effective than aspirin alone in decreasing the incidence of MI, stroke, and cardiovascular death after the initial visit. A greater incidence of bleeding is also noted, especially in those who undergo coronary bypass grafting within 5 days of therapy [23]. Clopidogrel has not been evaluated against placebo in any outcome study in the setting of primary PCI, but a higher loading dose of $600 \mathrm{mg}$ and maintenance dose of $150 \mathrm{mg}$ in the first week was superior to the $300 / 75 \mathrm{mg}$ regimen in the subset undergoing PCI in the Optimal Antiplatelet Strategy (OASIS) 7 trial. Use of high clopidogrel doses has been demonstrated to achieve more rapid inhibition on the ADP receptor [24].

(3) The preferred ADP receptor blockers-prasugrel or ticagrelol.

To date, no studies have evaluated the commencement of dual anti-platelet therapy prior to hospital admission, nor its use before, rather than during, angiography in the setting of STEMI.

Prasugrel is converted to its active metabolite more efficiently than clopidodrel and as such is more potent and has a more rapid onset. The Thrombolysis in Myocardial Infarction 38 (TRITON-TMI 38) study showed that prasugrel reduced the composite primary endpoint (cardiovascular 
non-fatal MI, or stroke) in clopidogrel-naïve patients who underwent coronary angiogram without significant increase non-CABG related bleeding risk [25]. Prasugrel is contraindicated in patients with prior stroke/transient ischaemic attack, and generally not recommended in patients aged $>75$ years or with lower body weight $(<60 \mathrm{~kg})$ as it was not associated with net clinical benefit in these subsets. The prasugrel prescription information stated that if patient is less than $60 \mathrm{~kg}$, similar loading dose but a reduced maintenance dose of $5 \mathrm{mg}$ is recommended. No outcome data is available with this dose. The 50th percentile weight (SD) of the male and female population in Singapore is $63 \mathrm{~kg} \mathrm{(6.89)}$ and $55 \mathrm{~kg}(6.73)$ respectively [26].

Ticagrelor binds reversibly to $\mathrm{P}_{2} \mathrm{Y}_{12}$ platelet ADP receptors. In the Platelet Inhibition and Patient Outcomes (PLATO) trial [27], Ticagrelor reduced the composite primary endpoint (cardiovascular death, non-fatal MI, or stroke) and also cardiovascular mortality in clopidogrelnaïve or pre-treated STEMI patients planned for primary PCI or moderate-to-high risk NSTE-ACS (planned for either conservative or invasive management). There was no significant difference in overall major bleeding rates between the clopidogrel and ticagrelor groups. In subset of patients with STEMI, the benefit was consistent. Ticagrelor may cause transient dyspnea at the onset of therapy, not associated with morphological or functional lung abnormalities. Ticagrelor can also be associated with asymptomatic bradycardia. None of the more potent agents (prasugrel or ticagrelor) should be used in patients with previous haemorrhagic stroke or moderate-to-severe liver disease. In these instances, clopidrogrel should be used.

Few clinical trials on intravenous glycoprotein IIb/IIIa in the management of STEMI were done after the era of routine double antiplatelet therapy. None of them showed that intravenous glycoprotein IIb/IIIa definitely improved clinical outcome [28]. A meta-analysis of randomised controlled trials of intracoronary versus intravenous administration of glycoprotein IIb/IIIa inhibitors during PCI for ACS showed that short term (1 month to 3 months) mortality was better with intracoronary glycoprotein IIb/ IIIa inhibitors but there was no difference in the medium or long term ( $\geq 6$ months) mortality [29]. There is no trial regarding the use of glycoprotein IIb/IIIa inhibitors with asprin plus the new and more potent oral anti-platelets eg prasugrel or ticagrelor.

Anti-thrombin Inhibitor (Anti-coagulant)

\section{Heparins}

UFH and low molecular weight heparin (LMWH) inactivate thrombin and activated factor $\mathrm{X}$ (factor $\mathrm{Xa}$ ). The AHA/ACC/ERC guidelines state that STEMI patients undergoing primary PCI should be given parenteral anticoagulants, although this has not been studied in placebocontrolled trials. In Singapore, UFH would be initiated in cardiac catheterisation laboratory during coronary angiogram and primary PCI. UFH should be given as IV bolus $60 \mathrm{u} / \mathrm{kg}$ (maximum 4,000 u) followed by initial infusion of $12 \mathrm{u} / \mathrm{kg} / \mathrm{h}$ (maximum 1,000 u/h). Activated partial prothrombin (aPTT) should be measured after $3 \mathrm{~h}$, and dose of UFH infusion should be adjusted to keep aPTT between 1.5 and 2 times the control value [30••]. UFH should be given intravenously in patients receiving reperfusion therapy with t-PA, r-PA, or TNK-tPA [31, 32], but evidence is lacking for non-selective fibrinolytics. LMWH has several advantages over UFH. The LMWH has a standard dose of administration, a lower incidence of heparin induced thrombocytopenia, and is easier to administer. There is also no need to monitor these patients. However, LMWH has a longer half life and is difficult to reverse.

Several clinical trials and one meta-analysis documented similar or improved composite outcomes (death, MI, and/ or recurrent angina or recurrent ischemia or revascularization) when enoxaparin was administered instead of UFH to patients with STEMI undergoing fibrinolysis [33]. Patients initially treated with enoxaparin should not be switched to UFH and vice versa because of increased risk of bleeding in patients $<75$ years the initial dose of enoxaparin is $30 \mathrm{mg}$ IV bolus followed by $1 \mathrm{mg} / \mathrm{kg}$ subcutaneous (SC) enoxaparin every $12 \mathrm{~h}$.

If the patients are $\geq 75$ years, enoxaparin dosage should be adjusted to $0.75 \mathrm{mg} / \mathrm{kg}$ ( $75 \%$ of normal dose) and IV bolus omitted. A study comparing enoxaparin with UFH in patients $\geq 75$ years undergoing fibrinolysis showed reduction in the primary endpoint (death and non-fatal recurrent MI) in the enoxaparin group. Using this dosage, patients with impaired renal function (creatinine clearance $<30 \mathrm{~mL} / \mathrm{min}$ ) may be given $1 \mathrm{mg} / \mathrm{kg}$ enoxaparin SC once daily [34•].

Fondaparinux selectively binds anti-thrombin and causes rapid and predictable inhibition of factor $\mathrm{Xa}$. It is chemically related to the LMWHs. A large trial concluded that in patients with STEMI treated with thrombolytic agent (73\% streptokinase), fondaparinux compared to placebo or UFH significantly reduces mortality and reinfarction without increasing bleeding and strokes. There was no benefit in STEMI patients undergoing PCI. There was a higher rate of guiding catheter thrombosis [35].

\section{Direct Anti-thrombin Inhibitors}

Bivalirudin is a specific direct thrombin inhibitor. It has a short half life of $25 \mathrm{~min}$ and does not cause heparin induced thrombocytopenia.

In STEMI patients undergoing PCI, anti-coagulation with bivalirudin alone, as compared with heparin plus 
glycoprotein IIb/IIa inhibition, resulted in significantly reduced 30-day rate of major bleeding and cardiac death [36]. The HERO-2 (Hirulog and Early Reperfusion or Occlusion) trial showed reduced reinfarction with intravenous bivalirudin compared to intravenous UFH in more than 17,000 patients who had streptokinase IV within $6 \mathrm{~h}$ of a STEMI [37]. It is reasonable for STEMI patients with heparin induced thrombocytopenia who are at a high risk of bleeding undergoing PCI or intravenous streptokinase to be administered bivalirudin. Bivalirudin has not been studied with fibrin-specific agents.

\section{Analgesia/Anti-ischemic Treatments}

Nitroglycerin (glyceryl tri-nitrate or GTN) produces benefits theoretically through

(a) Direct vasodilation of coronary arteries at the sites of plaque disruption,

(b) Dilatation of veins, which decreases pre-load,

(c) Dilatation of peripheral vessels with a resulting drop in systemic vascular resistance (afterload) and, therefore, myocardial oxygen workload demand.

For patients with chest pain with suspected ACS and systolic blood pressure of greater than $90 \mathrm{mmHg}$, a sublingual GTN tablet $(0.4 \mathrm{mg}) 5 \mathrm{~min}$ apart up to a maximum of three doses. If symptoms are not fully relieved and blood pressure is adequate, the patient may be started on intravenous GTN with initial infusion rates at $5 \mu \mathrm{g} / \mathrm{min}$ and titrate to a symptom-free state. The physician should increase the infusion at regular intervals, allowing a $10 \%$ reduction in the mean arterial pressure if normotensive and a $20-30 \%$ reduction if initially hypertensive. Usually complete benefit is achieved by $200 \mu \mathrm{g} / \mathrm{min}$. Extreme care must be exercised in patients with hypotension, inferior wall $\mathrm{AMI}$ and right ventricular (RV) infarction when administering GTN. GTN is contraindicated within $24 \mathrm{~h}$ of sildenafil (Viagra) use.

\section{Complication Following STEMI}

(1) Cardiogenic shock and acute left ventricle failure occurs if $40 \%$ or more of the left ventricular myocardium is infarcted. Primary PCI is preferred in this group of patients. In hospitals without PCI facilities, where transfer to PCI is delayed, fibrinolytic therapy should be initiated. Intra-aortic balloon pump (IABP) works synergistically with PCI or fibrinolytic agents.

(2) RV infarction. In patients with inferior MI, it is routine to record right sided ECG leads $\mathrm{V}_{4} \mathrm{R}$ to look for ST elevation to diagnose concomitant RV infarction. If the patient is hypotensive due to $\mathrm{RV}$ infarction, hemodynamics can be improved by judicious fluid challenge to augment ventricle pre-load and cardiac output. Venodilators, e.g. Nitrates are contraindicated. Early reperfusion is the goal.

(3) If a pre-systolic murmur is heard over the precordium by auscultation and the patient is in pulmonary edema or cardiogenic shock, an emergency echocardiography should be done to confirm the diagnosis of acute mitral valve regurgitation or rupture ventricle septum. Both groups of patient require intravenous diuretics, vasodilation/inotopic as well as IABP and ventilatory support. Early coronary angiography and cardiac surgery should be arranged.

(4) Arrhythmias. Ventricular premature beats and nonsustained ventricular tachycardia (lasting $<30 \mathrm{~s}$ ) do not require specific treatment. Ventricular fibrillation should be managed by immediate defibrillation and cardiopulmonary resuscitation according to Advanced Cardiac Life Support guidelines.

\section{Adjunctive Therapy to be Started not in the ED but Within the First $24 \mathrm{~h}$ of STEMI}

(1) $\beta$-Adrenergic blocking agents ameliorate the effects of catecholamine-induced tachycardia, decreased contractility and myocardial oxygen demand. A large retrospective study documented that AMI patients treated with $\beta$-blockers was associated with a $40 \%$ reduction in mortality. The $40 \%$ reduction was also seen in patients with conditions that are contraindicated to $\beta$-blockade use, e.g. congestive heart failure, chronic pulmonary disease, older age group [38]. Early administration of IV $\beta$-blockers was associated with cardiogenic shock from Day 0 and 1 after hospitalization. Long-term treatment with oral $\beta$-blockers should be initiated after patient has been stabilized.

(2) Angiotensin-converting enzyme (ACE) inhibitors prevent adverse left ventricular remodeling and have been shown to decrease cardiovascular mortality, delay deterioration in cardiac functional state and decrease incidence of sudden death and recurrent AMI development after the initial MI [39]. They are particularly helpful in patients with LV dysfunction or congestive heart failure, and in ACS patients with diabetes mellitus and large AMIs, especially in the anterior territory. The initial doses should be low in order to avoid hypotension. Once started, long-term use is generally the rule. Hypotension is the main contraindication to ACE inhibitor therapy. Relative contraindications include volume depletion and 
borderline perfusion. Close monitoring of renal function will be required during therapy.

(3) Meta-analysis showed that in post-MI patients, early high dose statin therapy reduced the risk of cardiovascular death, non-fatal MI, ischemia stroke.

\section{Conclusion}

Studies have shown an improvement of survival of STEMI due to increased use of perfusion therapy and recommended medication.

Review of the medical records of residents in the Worcester metropolitan area hospitalized at 11 greater Worcester medical centers for either STEMI or NSTEMI from 1997 to 2005 showed the 1-year post-discharge death rates of STEMI declined from 10.6 to $8.4 \%$ [40]. Similarly, data from four French nationwide registries documented that standardized 30-day mortality of STEMI decreased from $11.3 \%$ (95\% CI, 9.5-13.2) in 1995 to $4.4 \%$ (95\% CI, 3.5-5.4) in 2005 [41]. Multi-variable analysis showed that the change is accompanied by an increase in the proportion of women younger than 60 years with STEMI, as well as greater use of reperfusion therapy and recommended medications.

The success of timely reperfusion therapy for STEMI patients would require close collaboration between prehospital care, Emergency Medicine, hospitalist and Cardiology providers to address the processes and professional issues in the various stages of patient management.

Disclosure No potential conflicts of interest relevant to this article were reported.

\section{References}

Papers of particular interest, published recently, have been highlighted as:

- Of importance

•- Of major importance

1. Bernard R, Corday E, Eliasch $\mathrm{H}$, et al. Nomenclature and criteria for diagnosis of ischemic heart disease. Circulation. 1979;59(3): 607-9.

2. DeWood MA, Spores J, Notske R, et al. Prevalence of total coronary occlusion during the early hours of transmural myocardial infarction. N Engl Med. 1980;303:897-902.

3. DeWood MA, Stifter WF, Simpson Carroll S, et al. Coronary arteriographic findings soon after non-Q-wave myocardial infarction. N Eng J Med. 1986;315:417-23.

4. GISSI. Long-term effects of intravenous thrombolysis in acute myocardial infraction: final report of the GISSI study. Lancet. $1987 ; 871-74$
5. Arai AE, Hirsch GA. Q-wave and non-Q-wave myocardial infarctions through the eyes of cardiac magnetic resonance imaging. JACC. 2004;44(3):561-3.

6. Goodman SG, Langer A, Ross AM, et al. Non-Q-wave versus Q-wave myocardial infarction after thrombolytic therapy: angiographic and prognostic insights from the global utilization of streptokinase and tissue plasminogen activator for occluded coronary arteries-I angiographic substudy. Circulation. 1998; 97:444-50.

7. Wagner GS, Macfarlane P, Wellens H, et al. AHA/ACCF/HRS recommendations for the standardization and interpretation of the electrocardiogram. JACC. 2009;53(12):1003-11.

8. Yan AT, Yan RT, Kennelly BM, et al. Relationship of ST elevation in lead aVR with angiographic findings and outcome in non-ST elevation acute coronary syndrome. Am Heart J. 2007;154(1):71-8.

9. - Boersma E. Does time matter? A pooled analysis of randomized clinical trials comparing primary percutaneous coronary intervention and in-hospital fibrinolysis in acute myocardial infarction patients. Eur Heart J. 2006;27:779-88. Individual patient data from 22 trials $(n=6763)$ were pooled, and multi-level logistic regression assessed the relationship among treatment, treatment delay, and 30-day mortality. PPCI was associated with signifcantly lower 30-day mortality relative to FL, regardless of treatment delay.

10. Cooper RS, Simmons B, Castaner A, et al. Survival rates and prehospital delay during myocardial infarction among black persons. Am J Cardiol. 1986;57:208.

11. Herlitz J, Hartford M, Blohm M, et al. Effect of a media campaign on delay times and ambulance use in suspected acute myocardial infarction. Am J Cardiol. 1989;64:90-93.

12. Ong MEH, Wong ASL, Seet CM, et al.: Nationwide improvement of door-to-balloon times in patients with acute ST-segment elevation myocardial infarction requiring primary percutaneous coronary intervention with out-of-hospital 12-lead ECG recording and transmission. Ann Emerg Med. 26 Sep 2012. doi: 10.1016/j.annemergmed.2012.08.020.

13. Ong MEH, Wong ASL, Chan KP, et al. Improving door-to-door times in Asia for acute ST elevation myocardial infarction via direct activation by emergency physicians of percutaneous coronary intervention. Int J Cardiol. 2010;145(3):613-4.

14. - Andersen HR, Nielson TT, Rasmussen K, et al. A comparison of coronary angioplasty with fibrinolytic therapy in acute myocardial infarction. N Engl J Med. 2003;349(8):733-42. 1572 patients with acute myocardial infarction to treatment with angioplasty or accelerated treatment with intravenous alteplase. The primary study endpoint (a composite of death, clinical evidence of reinfarction, or disabling stroke at 30 days) was reached in 8.5 percent of the patients in the angioplasty group, as compared with 14.2 percent of those in the fibrinolysis group $(P=0.002)$

15. •• Keeley EC, Boura JA, Grines CL. Primary angioplasty versus intravenous thrombolytic therapy for acute myocardial infarction: a quantitative review of 23 randomized trial. Lancet. 2003;361:13-20. A review of 23 trials, which together randomly assigned 7739 thrombolytic-eligible patients with ST-segment elevation AMI to primary PTCA $(n=3872)$ or thrombolytic therapy $(n=3867)$ primary PTCA was better than thrombolytic therapy at reducing overall short-term death $(7 \%[n=270] \mathrm{vs}$ $9 \%$ [360]; $p=0.0002)$, death excluding the SHOCK trial data (5\% [199] vs $7 \%$ [276]; $p=0.0003)$, non-fatal reinfarction (3\% [80] vs $7 \%$ [222]; $p<0.0001)$, stroke (1\% [30] vs $2 \%$ [64]; $p=0.0004)$, and the combined endpoint of death, non-fatal reinfarction, and stroke (8\% [253] vs $14 \%$ [442]; $p<0.0001$ ). The results seen with primary PTCA remained better than those seen with thrombolytic therapy during long-term follow-up, and 
were independent of both the type of thrombolytic agent used, and whether or not the patient was transferred for primary PTCA.

16. • O'Conor RE, Brady W, Brooks SC, et al. Part 10 acute coronary syndrome: 2010 American Heart Association guidelines for cardiopulmonary resuscitation and emergency cardiovascular care. Circulation. 2010;122(suppl 3):S787-817.

17. Lemay MR, Davies RF, Dionne R, et al. Comparison of early mortality of paramedic-diagnosed ST-segment elevation myocardial infarction with immediate transport to a designated primary percutaneous coronary intervention center to that of similar patients transported to the nearest hospital. Am J Cardiol. 2006;98;1329-33.

18. Pinto DS, Kirtane AJ, Nallamothu BK, et al. Hospital delays in reperfusion for ST-elevation myocardial infarction: implications when selecting a reperfusion strategy. Circulation. 2006;114: 2019-25.

19. ${ }^{\bullet}$ Steg G, James SK, Dan A, et al. ESC guidelines for the management of acute myocardial infarction in patients presenting with ST-segment elevation. Eur Heart J. 2012;33:2569-619. The latest guidelines developed by the European Society of Cardiology (ESC) on the management of acute ST elevation Myocardial Infarcts. This includes prehospital services, reperfusion therapies as well as pharmacological therapy. It also covers the management of the complications arising from ST elevation myocardial infarcts.

20. A comparison of reteplase with alteplase for acute myocardial infarction. The Global Use of Strategies to Open Occluded Coronary Arteries (GUSTO III) Investigators. N Engl J Med. 1997;337(16):1118-23.

21. Assessment of the Safety and Efficacy of a New Thrombolytic (ASSENT) Investigators. Single-bolus tenecteplase compared with front-loaded alteplase in acute myocardial infarction: the ASSENT-2 double-blind randomized trial. Lancet. 1999;354(9180): 716-22.

22. ISIS-2 (Second International Study of Infarct Survival) Collaborative Group. Randomised trial of intravenous streptokinase, oral aspirin, both or neither among 17,187 cases of suspected acute myocardial infarction: ISIS-2. Lancet. 1998;3(9):349-60.

23. Sabatine MS, Cannon CP, Gibson M, et al. Addition of clopidogrel to aspirin and fibrinolytic therapy for myocardial infarction with ST-segment elevation. N Engl J Med. 2005;352 (12):1179-89.

24. The CURRENT-OASIS 7 Investigators. Dose comparisons of clopidogrel and aspirin in acute coronary syndromes. N Engl $\mathrm{J}$ Med. 2010;363:930-42.

25. Wiviott SD, Braunwald E, McCabe $\mathrm{CH}$, et al. Prasugrel versus clopidogrel in patients with acute coronary syndromes. N Engl J Med. 2007;357(20):2001-15.

26. Tan KC, Hartono M, Kumar N. Anthropometry of the Singaporean and Indonesian populations. Int J Ind Ergon. 2010;40: 757-66.

27. Wallentin L, Becker RC, Budaj A, et al. Ticagrelor versus clopidogrel in patients with acute coronary syndromes. N Engl J Med. 2009;361(11):1045-57.

28. Thiele H, Wöhrle J, Rittger H, et al. Intracoronary versus intravenous bolus abciximab during primary percutaneous coronary intervention in patients with acute ST-elevation myocardial infarction: a randomised trial. Lancet. 2012;379:923-31.

29. Friedland S, Eisenberg MJ, Shimony A. Meta-analysis of randomized controlled trials of intracoronary versus intravenous administration of glycoprotein IIb/IIIa inhibitors during percutaneous coronary intervention for acute coronary syndrome. Am J Cardiol. 2011;108:1244-51.

30. • Kushner FG, Hand M, Smith SC et al. 2009 Focused updates: ACC/AHA guidelines for the management of patient with STelevation myocardial infarction: (updating the 2004 guideline and
2007 focused update) and ACC/AHA/SCAI guidelines on percutaneous coronary intervention (updating the 2005 guideline and 2007 focused update): a report of the American College of Cardiology Foundation/American Heart Association Task Force on Practice ACC/AHA/SCAI guidelines on percutaneous coronary intervention (updating the 2005 guideline and 2007 focused update): a report of the American College of Cardiology Foundation/American Heart Association Task Force on Practice Guidelines. Circulation. 2009;120:2271-306. doi: 10.1161/ CIRCULATIONAHA.109.192663. These are the most recent guidelines published by the AHA/ACC/SCAI.

31. ISIS-3 (Third International Study of Infarct Survival) Collaborative Group. ISIS-3: a randomized comparison of streptokinase vs tissue plasminogen activator vs anistreplase and of aspirin plus heparin vs aspirin alone among 41,299 cases of suspected acute myocardial infarction. Lancet. 1992;339(8796):753-70.

32. Gruppo Italiano per lo Studio della Sopravvivenza nell'Infarto Miocardico. GISSI-2: a factorial randomized trial of alteplase versus streptokinase and heparin versus no heparin among 12,490 patients with acute myocardial infarction. Lancet. 1990;336: $65-71$.

33. Wallentin L, Goldstein P, Armstrong PW, et al. Efficacy and safety of tenecteplase in combination with the low-molecularweight heparin enoxaparin or unfractionated heparin in the prehospital setting: the Assessment of the Safety and Efficacy of a New Thrombolytic Regimen 9 (ASSENT)-3 PLUS randomized trial in acute myocardial infarction. Circulation. 2003;108: 135-42.

34. •Antman EM, Morrow DA, McCabe CH, et al. Enoxaparin versus unfractionated heparin with fibrinolysis for ST-elevation myocardial infarction. N Engl J Med. 2006;354(14):1477-88. 20,506 Patients with ST-elevation myocardial infarction who were scheduled to undergo fibrinolysis to receive enoxaparin throughout the index hospitalization or weight-based unfractionated heparin for at least $48 \mathrm{~h}$. In patients receiving fibrinolysis, treatment with enoxaparin throughout the index hospitalization is superior to treatment with unfractionated heparin for $48 \mathrm{~h}$ but is associated with an increase in major bleeding episodes.

35. Yusuf S, Mehta SR, Chrolavicius S, et al. Effects of fondaparinux on mortality and reinfarction in patients with acute ST-segment elevation myocardial infarction: the OASIS- 6 randomized trial. JAMA. 2006;295(13):1519-30.

36. Stone GW, Witzenbichler B, Guagliumi G, et al. Bivalirudin during primary PCI in acute myocardial infarction. N Engl J Med. 2008;358(21):2218-30.

37. White $\mathrm{H}$, for the Hirulog and Early Reperfusion or Occlusion (HERO)-2 Trial Investigators. Thrombin-specific anticoagulation with bivalirudin versus heparin in patients receiving fibrinolytic therapy for acute myocardial infarction: the HERO-2 randomised trial. Lancet. 2001;358:1855-63.

38. Gottlieb SS, McCarter RJ, Vogel RA. Effect of beta-blockade on mortality among high-risk and low-risk patients after myocardial infarction. N Engl J Med. 1998;339(8):489-97.

39. ISIS-4: a randomized factorial trial assessing early oral captopril, oral mononitrate, and intravenous magnesium sulphate in 58050 patients with suspected acute myocardial infarction. Lancet. 1995; 345:669-85.

40. McManus DD, Gore JJ, Yarzebski J, et al. Recent trends in the incidence, treatment, and outcomes of patients with ST and nonST-segment acute myocardial infarction. Am J Med. 2011;124(1):40-7. doi:10.1016/j.annemergmed.2010.08.020.

41. Puymirat E, Simon T, Steg PG, et al. Association of changes in clinical characteristics and management with improvement in survival among patients with ST-elevation myocardial infarction. JAMA. 2012;308(10):998-1006. 